\title{
Multiple Colorectal Mucosa- Associated Lymphoid Tissue Lymphoma Successfully Treated with Chemotherapy
}

\author{
Makoto Saito $^{a}$ Shihori Tsukamoto ${ }^{a}$ Takashi Ishio $^{a} \quad$ Emi Yokoyama ${ }^{a}$ \\ Koh Izumiyama $^{a}$ Akio Moria Masanobu Morioka ${ }^{a}$ Takeshi Kondo ${ }^{a}$ \\ Hirokazu Sugino ${ }^{\text {b }}$ \\ aDepartment of Internal Medicine and Hematology/Blood Disorders Center, Aiiku Hospital, \\ Sapporo, Japan; ${ }^{b}$ Department of Cancer Pathology, Faculty of Medicine, Hokkaido \\ University, Sapporo, Japan
}

\author{
Keywords \\ Colorectal lymphoma - Mucosa-associated lymphoid tissue lymphoma - Treatment . \\ Chemotherapy
}

\begin{abstract}
The standard treatment for colorectal mucosa-associated lymphoid tissue (MALT) lymphoma has not yet been established due to the rarity of the disease. Here, we report a case of longterm response to chemotherapy for colorectal MALT lymphoma (stage I). A 77-year-old frail female patient with diabetes mellitus and dementia developed melena of unknown etiology, and a colonoscopy was performed at a nearby hospital. A biopsy suggested malignant lymphoma, and she was referred to our department. As a result of re-examination of colonoscopy, a total of 3 submucosal tumor-like lesions were confirmed. Of these, a biopsy of the lesions in the ascending colon and rectum was performed, and MALT lymphoma was diagnosed on the basis of the histopathological findings. Following close examination, no other lymphoma lesions were found, and the patient was diagnosed with primary colorectal MALT lymphoma, stage I. After 1 course of R-THP-COP chemotherapy (rituximab + cyclophosphamide, pirarubicin, vincristine, and prednisone), the rectal lesion was confirmed to have almost disappeared endoscopically, and lymphoma cells were not found histopathologically. The patient was determined to be in complete remission (CR). However, due to hematological toxicity and a slight worsening of glucose control, the second chemotherapy course was changed
\end{abstract}

\section{Karger $\stackrel{2}{*}$}


to the BR regimen (rituximab + bendamustine), and 4 courses were performed ( 5 total courses of chemotherapy). Currently, $>3$ years have passed since reaching $C R$, and the patient is alive without recurrence.

\section{Introduction}

Mucosa-associated lymphoid tissue (MALT) lymphoma, derived from the follicular marginal zone of MALT, occurs in various organs, mainly in the stomach [1]. MALT lymphoma grows very slowly in any organ and is generally considered to have a good prognosis. Standard treatment for primary gastric MALT lymphoma, such as eradication therapy for Helicobacter pylori-positive cases, has already been established [2]. However, colorectal MALT lymphoma is rare, and standard treatment has not yet been established. Here, we report a long-term response to chemotherapy in a patient with multiple colorectal MALT lymphoma lesions.

\section{Case Presentation}

A 77-year-old frail female patient with diabetes mellitus and dementia visited a nearby hospital for bloody stool of unknown etiology. Colonoscopy revealed 3 abnormal lesions in the colorectum. Biopsy suggested malignant lymphoma, so the patient was referred to our department and hospitalized. Laboratory data at admission (Table 1) showed mild anemia, with $\mathrm{Hb} 11.3$ (normal range 12.0-16.0) g/dL, and mild thrombocytosis, with 44.7 (12.0-40.0) $\times 10^{4} / \mu \mathrm{L}$. Serum $H$. pylori antibody was negative, and soluble interleukin- 2 receptor and serum ferrum were normal. In addition, fasting blood glucose was $148 \mathrm{mg} / \mathrm{dL}$, and HbA1c was high at 7.8\%. Colonoscopy revealed submucosal tumor-like lesions in a total of 3 locations: the ascending colon (1.5 $\mathrm{cm}$ diameter), the sigmoid colon (5 $\mathrm{mm}$ diameter), and the rectum (2-2.5 cm diameter) (Fig. 1). Of these, the lesions of the ascending colon and rectum had shallow ulcered surfaces, consistent with malignant lymphoma, especially diffuse large B-cell lymphoma (DLBCL). The ascending colon and rectal lesions were biopsied.

Table 1. Laboratory findings at admission

\begin{tabular}{|c|c|c|c|c|c|}
\hline \multicolumn{2}{|c|}{ Blood count } & \multicolumn{2}{|c|}{ Biochemistry } & \multicolumn{2}{|c|}{ Serological examination } \\
\hline WBC & $7,500 / \mu \mathrm{L}$ & $\mathrm{TP}$ & $7.9 \mathrm{~g} / \mathrm{dL}$ & CRP & $(-), 0.07 \mathrm{mg} / \mathrm{dL}$ \\
\hline Neut & $77 \%$ & Alb & $4 \mathrm{~g} / \mathrm{dL}$ & IL-2R & $264 \mathrm{U} / \mathrm{mL}$ \\
\hline Lym & $18 \%$ & T.Bil & $0.4 \mathrm{mg} / \mathrm{dL}$ & H. pylori-IgG & $(-),<3 \mathrm{U} / \mathrm{mL}$ \\
\hline Mon & $5 \%$ & GOT & $19 \mathrm{IU} / \mathrm{L}$ & $\mathrm{s}-\mathrm{Fe}$ & $56 \mu \mathrm{g} / \mathrm{dL}$ \\
\hline $\mathrm{RBC}$ & $407 \times 10^{4} / \mu \mathrm{L}$ & GPT & $12 \mathrm{IU} / \mathrm{L}$ & & \\
\hline $\mathrm{Hb}$ & $11.3 \mathrm{~g} / \mathrm{dL}$ & LDH & $210 \mathrm{IU} / \mathrm{L}$ & Blood glucose & \\
\hline Hct & $34.1 \%$ & $\gamma$-GTP & $21 \mathrm{IU} / \mathrm{L}$ & FBS & $148 \mathrm{mg} / \mathrm{dL}$ \\
\hline MCV & $84 \mathrm{FL}$ & BUN & $10.3 \mathrm{mg} / \mathrm{dL}$ & HbA1c & $7.8 \%$ \\
\hline \multirow[t]{2}{*}{ Plt } & $44.7 \times 10^{4} / \mu \mathrm{L}$ & $\mathrm{Cr}$ & $0.79 \mathrm{mg} / \mathrm{dL}$ & & \\
\hline & & UA & $3.3 \mathrm{mg} / \mathrm{dL}$ & & \\
\hline
\end{tabular}

IL-2R, interleukin-2 receptor; s-Fe, serum ferrum. 


\section{Case Reports in Oncology}

\begin{tabular}{l|l}
\hline Case Rep Oncol 2021;14:1761-1767 \\
\hline DOI: 10.1159/000520428 & $\begin{array}{l}\text { @ 2021 The Author(s). Published by S. Karger AG, Basel } \\
\text { www.karger.com/cro }\end{array}$ \\
\hline
\end{tabular}

Saito et al.: Colorectal MALT Lymphoma Successfully Treated with Chemotherapy
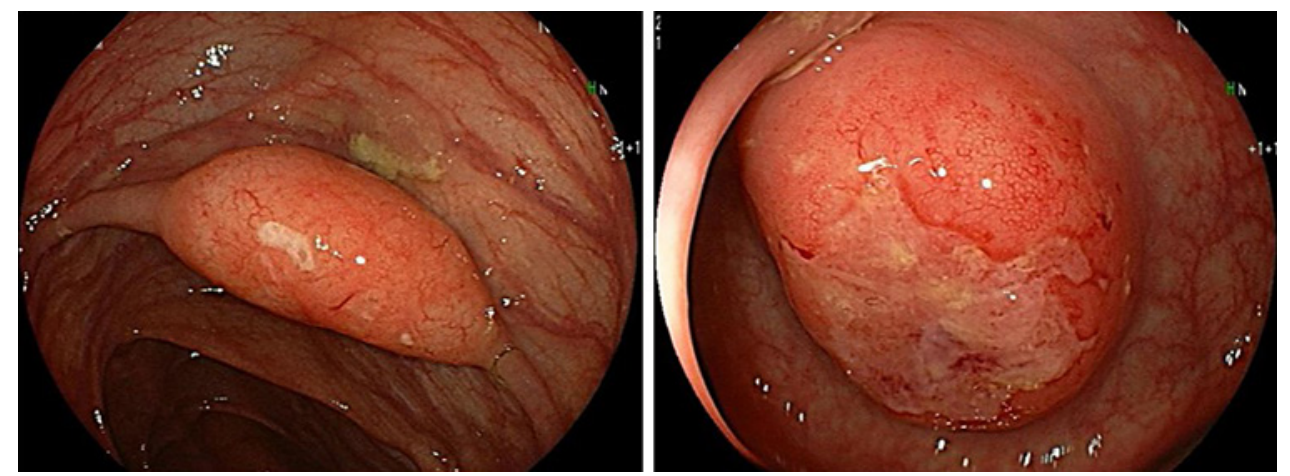

Fig. 1. Colonoscopic findings. (left) 1.5 -cm diameter lesion in the ascending colon, (right) 2- to 2.5-cm diameter lesion in the rectum. Submucosal tumor-like lesions with shallow ulcered surfaces were observed.
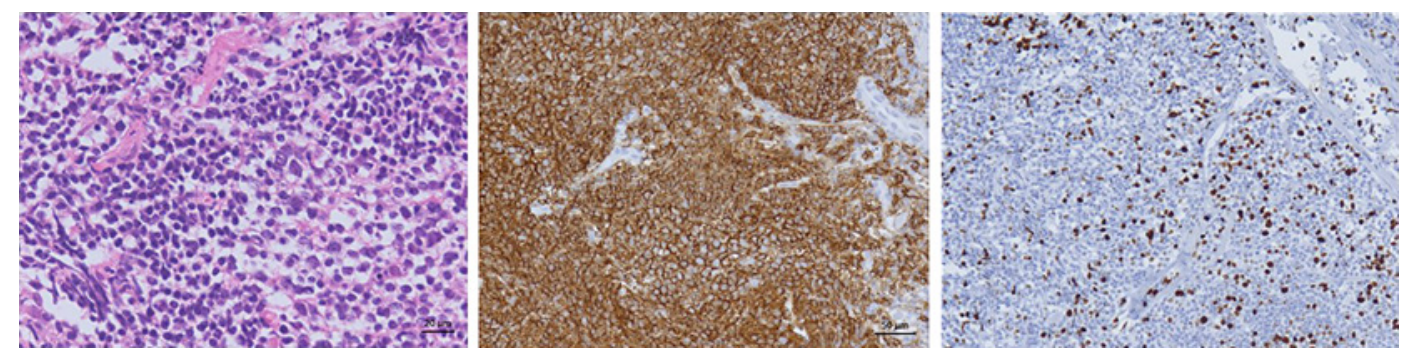

Fig. 2. Histopathological findings. (left) (HE staining) Small- to medium-sized abnormal lymphocytes were uniformly distributed; (middle) CD20 staining was positive; (right) the MIB-1 index was 20-30\%. These findings were consistent with MALT lymphoma. MALT, mucosa-associated lymphoid tissue.

Histopathologically, small- to medium-sized abnormal lymphocytes were uniformly distributed, CD20 was positive, and the MIB-1 index was 20-30\% (Fig. 2). IgH-BCL2, BCL6, and MALT1 were negative in fluorescence in situ hybridization. Although no lymphoepithelial lesion was observed, the pathological diagnosis was consistent with MALT lymphoma. Esophagogastroduodenoscopy showed no notable abnormalities, and contrast-enhanced CT of the whole body revealed no colorectal lesions and no other obvious abnormalities. FDG-PET/CT examination showed uptake in 2 regions, the ascending colon and the rectum, and the maximum standardized uptake value (SUVmax) was 5.5 in the ascending colon and 8.1 in the rectum (Fig. 3). No other abnormal uptake was found. Bone marrow aspiration showed no abnormal lymphocytes including a cell surface marker, PCR did not show IgH gene rearrangement, and the involvement of lymphoma cells was ruled out. On the other hand, the sample showed hyperplastic marrow (the number of nucleated cells was approximately $20 \times 10^{4} / \mu \mathrm{L}$ ), and myeloblasts were slightly abundant (in the $1 \%$ range). Chromosomal abnormalities $46, \mathrm{XX}$, del (9) (q11q22) were observed. She was diagnosed with myelodysplastic syndrome.

Clinical course: based on the above findings, the patient was diagnosed with primary colorectal MALT lymphoma (stage I). First-line treatment was chemotherapy, and R-THPCOP (a regimen in which doxorubicin in CHOP was changed to pirarubicin, which is less cardiotoxic; rituximab $375 \mathrm{mg} / \mathrm{m}^{2}$ + cyclophosphamide $750 \mathrm{mg} / \mathrm{m}^{2}$, pirarubicin $50 \mathrm{mg} / \mathrm{m}^{2}$, vincristine $1.4 \mathrm{mg} / \mathrm{m}^{2}$, day 1 , and prednisone $60 \mathrm{mg}$, days 1-5) was administered. Upon colonoscopic re-examination, the rectal lesion had almost disappeared (Fig. 4), and no lymphoma cells were found in a histopathological analysis of the biopsy sample. However, grade 4 hematological toxicity according to the Common Terminology Criteria for Adverse Events (CTCAE) 


\section{Case Reports in Oncology}

Case Rep Oncol 2021;14:1761-1767

\begin{tabular}{l|l}
\hline DOI: $10.1159 / 000520428$ & $\odot 2021$ The Author(s). Published by S. Karger AG, Basel \\
\hline
\end{tabular} www.karger.com/cro

Saito et al.: Colorectal MALT Lymphoma Successfully Treated with Chemotherapy
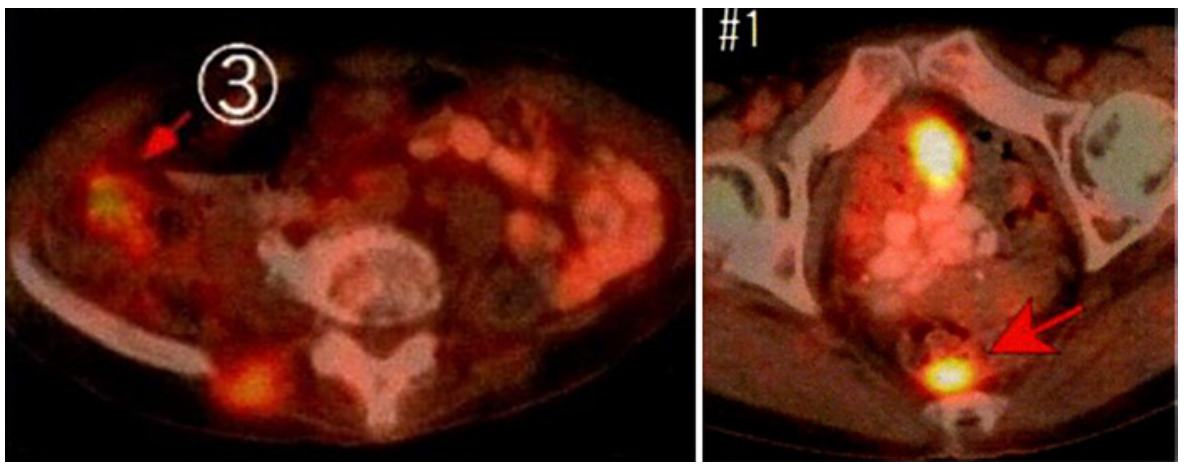

Fig. 3. PET-CT findings. Clear uptake was observed in 2 regions, (left) the ascending colon (SUVmax 5.5) and (right) the rectum (SUVmax 8.1).

Fig. 4. Colonoscopic findings in the rectum. After 1 course of R-THP-COP chemotherapy, the MALT lymphoma lesion disappeared. MALT, mucosa-associated lymphoid tissue.

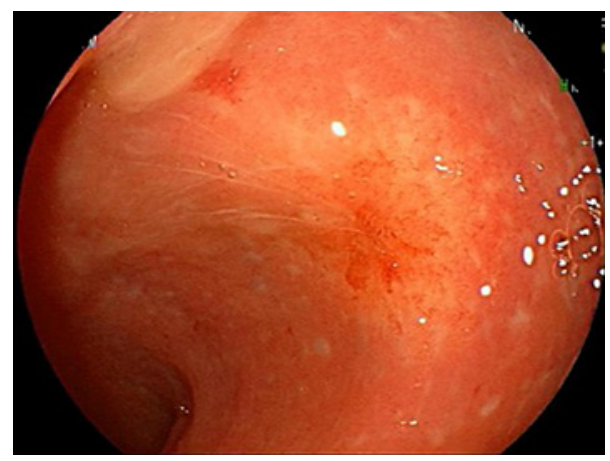

Version 5.0 developed, including a decrease in the white blood cell (WBC) count to $400 / \mu \mathrm{L}$ and a decrease in the neutrophil count to $32 / \mu \mathrm{L}$. In addition, blood glucose control showed a slightly worsened tendency, with a fasting blood glucose of $170-200 \mathrm{mg} / \mathrm{dL}$. Owing to the concern that the risk of infection would increase if the THP-COP regimen was repeated, the second chemotherapy course was changed to the BR regimen (rituximab $375 \mathrm{mg} / \mathrm{m}^{2}+$ bendamustine $90 \mathrm{mg} / \mathrm{m}^{2}$, days 1-2, every 4 weeks), and 4 courses were administered ( 5 chemotherapy courses in total). Skin eruption (grade 2-3 according to the CTCAE) due to bendamustine administration was observed after the final course, but it resolved with the use of a steroid preparation. Colonoscopy and PET/CT were re-performed, and both confirmed the disappearance of all lesions. The patient was determined to be in complete remission (CR) and was discharged. She was followed up as an outpatient with no treatment for MALT lymphoma, and although pneumonia and a worsening of dementia were observed during the follow-up, she recovered from both. Currently, 3 years and 8 months after reaching CR, she is alive without recurrence.

\section{Discussion}

The colon and rectum are the least common gastrointestinal locations for primary lymphoma, accounting for $<1 \%$ of all large-bowel malignancies and $\sim 10 \%$ of gastrointestinal lymphomas [3]. Colorectal MALT lymphoma is an even rarer disease [4]. Since the tumor cells of colorectal MALT lymphoma show a strong proliferative tendency deeper in the submucosa, it often exhibits features of a subepithelial (/submucosal) tumor type endoscopically [4] as 
in this case, and lymphoepithelial lesion, which is an infiltration of lymphoma cells into the epithelium, is often less noticeable. On the other hand, when DLBCL presents with submucosal tumor-like elevated lesions in the gastrointestinal tract, it is characterized by erosion or shallow ulcers on the mucosal surface [5]. This finding was seen in this patient, so DLBCL was initially suspected. Pathological findings consistent with MALT lymphoma were found in the biopsy specimen, but the possibility of DLBCL components in the deep mucosa of rectal tumor could not be ruled out. Recently, it was reported that the lesions in 26 of 27 patients with extragastric MALT lymphoma having a Ki-67 labeling (MIB-1) index exceeding 15\% could be detected by FDG-PET [6]. In this patient, the MIB-1 index was 20-30\%, and PET detected 2 out of 3 lymphoma lesions. These lesions were not detected by PET after the final course of chemotherapy, and PET was as useful in assessing therapeutic efficacy as colonoscopy. In particular, the SUVmax of the rectal lesion was 8.1, suggesting that the growth of the tumor was rather rapid and that the tumor may have a higher potential of malignancy.

According to the Japanese practical guidelines for hematological malignancies (2018, revised version) [7], careful follow-up without therapy, irradiation, and surgical resection are recommended for the treatment of extragastric MALT lymphoma in the localized stage. As local treatments, the effectiveness of surgical or endoscopic tumor resection and irradiation has been reported for primary colorectal MALT lymphoma [8, 9]; however, follow-up without treatment may be acceptable when patients are asymptomatic. As a drug treatment, the usefulness of $H$. pylori eradication [10] and anticancer drug treatment, including rituximab (RTX) monotherapy [11], has also been reported. Thus, there is still no established standard treatment for primary colorectal MALT lymphoma. Recently, review studies on colorectal MALT lymphoma, including its treatment and prognosis, have been reported $[4,12,13]$. According to Won et al. [12], among 73 patients, excluding 2 untreated patients, 71 received first-line treatment, including surgery (19), local resection (19), chemotherapy (13), radiation therapy (5), and antibiotic therapy, including H. pylori eradication (15). Remission rates were all high, with no difference among patients treated with tumor resection, chemotherapy, radiation therapy, and antibiotics $(92.1 \%$ vs. $92.3 \%$ vs. $80 \%$ vs. $80 \%, p=0.29$ ) [12]. In the review by Jeon et al. [4], among 51 patients, endoscopic mucosal resection (EMR) (17), surgery (8), radiation (12), chemotherapy (4), EMR + radiation (4), and surgery + radiation (1) were performed, and the authors concluded that the prognosis of colorectal MALT lymphoma was excellent, including the prognoses of 5 untreated patients [13]. There are a variety of chemotherapy regimens in addition to RTX monotherapy, including CHOP ( \pm RTX), chlorambucil ( $\pm \mathrm{RTX}$ ), cyclophosphamide (monotherapy), MACOPB (methotrexate with leucovorin rescue, doxorubicin, cyclophosphamide, vincristine, prednisone, and bleomycin), and BR $[11,12]$.

Our patient was already bleeding, and the treatment had to be administered quickly. Surgical or endoscopic tumor resection was considered to be difficult because there were 3 distant lymphoma lesions in the large intestine from the ascending colon to the rectum. Radiation therapy was also impossible because the irradiation field was too wide. In addition, as mentioned above, our patient was considered to have MALT lymphoma with a higher potential of malignancy, and there was concern that MALT lymphoma might transform into DLBCL. Therefore, instead of $H$. pylori eradication therapy or RTX monotherapy, we chose chemotherapy. The treatment was administered according to the Japanese guideline [7] mentioned above, which recommends a standard dose of R-CHOP for elderly DLBCL patients under the age of 80 , and without a dose reduction. As a result, the treatment was effective, and CR of the main lesion was reached at the end of 1 course of chemotherapy. However, this elderly patient had myelodysplastic syndrome, DM, and frailty, and grade 4 hematologic toxicity, namely, severe WBC and neutrophil count reductions $(400 / \mu \mathrm{L}$ and $32 / \mu \mathrm{L}$, respectively), developed

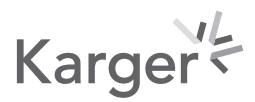


after treatment. Concerned about the complications of severe infections during chemotherapy, the second course was changed to the BR regimen, which is less intense. In indolent nonHodgkin lymphoma, it has been reported that BR was significantly better in progression-free survival than R-CHOP [14], and it was also reported that BR showed long-term efficacy and safety for MALT lymphoma [15]. Only 1 case of the BR regimen use was reported for colorectal MALT lymphoma [11], and it was used as a third-line treatment, but its therapeutic effect was unknown. Here, our patient received R-THP-COP chemotherapy as initial treatment, and after reaching $\mathrm{CR}$, she was treated with the $\mathrm{BR}$ regimen as consolidation-like treatment, which we determined to be effective. In the future, it will be necessary to consider whether chemotherapy with a BR regimen for remission is one of the treatment options for colorectal MALT lymphoma.

\section{Conclusion}

Here, we reported a case in which chemotherapy (R-THP-COP $\times 1$ course $+\mathrm{BR} \times 4$ courses) was effective in an elderly frail patient with 3 MALT lymphoma lesions in the large intestine. Standard treatment for colorectal MALT lymphoma has not yet been established due to its rarity. Currently, R-CHOP is widely used as chemotherapy for colorectal MALT lymphoma. It is necessary to validate whether BR, which has recently shown long-term efficacy and safety in the treatment of MALT lymphoma regardless of the affected organ, is also one of the treatment options for colorectal MALT lymphoma.

\section{Statement of Ethics}

The research presented in this manuscript was ethically conducted in accordance with the World Medical Association Declaration of Helsinki; the patient received the standard medical care without using any special protocol. Our institution does not require ethics committee approval for reporting individual cases or case series ( $<10$ patients). Written informed consent was obtained from the patient for publishing this article.

\section{Conflict of Interest Statement}

The authors declare no potential conflicts of interest in this work.

\section{Funding Sources}

No funding was received nor utilized in the production of this publication.

\section{Author Contributions}

Sugino H. was involved in the pathological procedure. All authors made substantial contributions to conception and design, acquisition of data, or analysis and interpretation of data; took part in drafting the article or revising it critically for important intellectual content; gave final approval of the version to be published; and agree to be accountable for all aspects of the work.

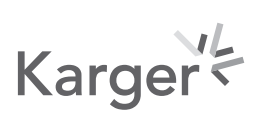




\section{Data Availability Statement}

All data relevant to the case presentation are available as part of the article, and no additional source data are required.

\section{References}

1 Cook JR, Isaacson PG, Chott A, Nakamura S, Müller-Hermelink HK, Harris NL, et al. Extranodal marginal zone lymphoma of mucosa-associated lymphoid tissue (MALT lymphoma). In: Swerdlow SH, Campo E, Harris NL, Jaffe ES, Pileri SA, Stein H, et al., editors. WHO classification of tumours of haematopoietic and lymphoid tissues. Lyon: IARC press; 2017. p. 259-62.

2 Nakamura S, Sugiyama T, Matsumoto T, Iijima K, Ono S, Tajika M, et al. Long-term clinical outcome of gastric MALT lymphoma after eradication of Helicobacter pylori: a multicentre cohort follow-up study of 420 patients in Japan. Gut. 2012 Apr;61(4):507-13.

3 Gay ND, Chen A, Okada CY. Colorectal lymphoma: a review. Clin Colon Rectal Surg. 2018 Sep;31(5):309-16.

4 Jeon MK, So H, Huh J, Hwang HS, Hwang SW, Park SH, et al. Endoscopic features and clinical outcomes of colorectal mucosa-associated lymphoid tissue lymphoma. Gastrointest Endosc. 2018 Feb;87(2):529-39.

5 Nakamura S, Matsumoto T. Diagnosis and management of gastrointestinal lymphomas. Gastroenterol Endosc. 2014;56(10):3599-606. (in Japanese).

6 Albano D, Bosio G, Giubbini R, Bertagna F. 18F-FDG PET/CT and extragastric MALT lymphoma: role of Ki-67 score and plasmacytic differentiation. Leuk Lymphoma. 2017 Oct;58(10):2328-34.

7 Extranodal marginal zone lymphoma of mucosa associated lymphoid tissue: MALT lymphoma/nodal marginal zone lymphoma/splenic marginal zone lymphoma. Japanese Society of Hematology practical guidelines for hematological malignancies (2018, revised version). Tokyo: Kanehara publishing; 2020. p. 190-207.

8 Wei YL, Min CC, Ren LL, Xu S, Chen YQ, Zhang Q, et al. Laterally spreading tumor-like primary rectal mucosaassociated lymphoid tissue lymphoma: a case report. World J Clin Cases. 2021 Jun 6;9(16):3988-95.

9 Kobayashi T, Takahashi N, Hagiwara Y, Tamaru J, Kayano H, Jin-nai I, et al. Successful radiotherapy in a patient with primary rectal mucosa-associated lymphoid tissue lymphoma without the API2-MALT1 fusion gene: a case report and review of the literature. Leuk Res. 2008 Jan;32(1):173-5.

10 Matsumoto T, Iida M, Shimizu M. Regression of mucosa-associated lymphoid-tissue lymphoma of rectum after eradication of Helicobacter pylori. Lancet. 1997 Jul 12;350(9071):115-6.

11 Tannoury J, Amiot A, Lemonnier F, Dupuis J, Gagnière C, Belhadj K, et al. Colonic mucosa-associated lymphoid tissue lymphoma: a case series. Leuk Lymphoma. 2020 Mar;61(3):582-7.

12 Won JH, Kim SM, Kim JW, Park JH, Kim JY. Clinical features, treatment and outcomes of colorectal mucosaassociated lymphoid tissue (MALT) lymphoma: literature reviews published in English between 1993 and 2017. Cancer Manag Res. 2019 Sep 20;11:8577-87.

13 Hollie N, Asakrah S. MALT lymphoma of the colon: a clinicopathological review. J Clin Pathol. 2020 Jul;73(7): 378-83.

14 Flinn IW, van der Jagt R, Kahl B, Wood P, Hawkins T, MacDonald D, et al. First-line treatment of patients with indolent non-Hodgkin lymphoma or mantle-cell lymphoma with bendamustine plus rituximab versus R-CHOP or R-CVP: results of the BRIGHT 5-year follow-up study. J Clin Oncol. 2019 Apr 20;37(12):984-91.

15 Salar A, Domingo-Domenech E, Panizo C, Nicolás C, Bargay J, Muntañola A, et al. Long-term results of a phase 2 study of rituximab and bendamustine for mucosa-associated lymphoid tissue lymphoma. Blood. 2017 Oct 12;130(15):1772-4. 